\title{
Tests of a scintillating-fibre tracking detector based on position-sensitive photomultiplier readout
}

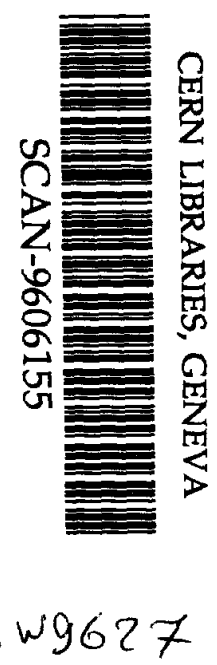

\author{
F. Bosi;, A. Cardini; $\quad$ V. Cavasinni; $\quad$ B. Di Girolamo; \\ S.I. Dolinsky ${ }^{\dagger} \quad$ V. Flaminio; $\quad$ A.M. Gorin; \\ K.K. Kuroda, I.V. Manuilov; A. Penzo, D. Rizzi*, \\ R. Ruberti; A. A. Salnikov ${ }^{\dagger} \quad$ M. Tareb-Reyes
}

\begin{abstract}
We report the results of measurements performed using a tracking detector based on $835 \mu \mathrm{m}$ scintillating fibres read-out by two positionsensitive photomultipliers. A six-layer hodoscope consisting of $1.2 \mathrm{~m}$ long fibres has been built and tested using cosmic rays. Subsequentely, two such hodoscopes have been assembled and tested using a hadron beam at the CERN PS. The efficiency per plane is found to be about $70 \%$ and the point resolution about $130 \mu \mathrm{m}$. The signal-to-noise ratio is about 2.4 and is dominated by crosstalk in the position-sensitive photomultipliers.
\end{abstract}

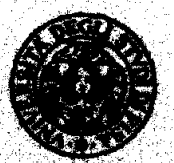

UNIVERSTTA DEGLI STUDI DI PISA DIPARTIMIANTO DI FIBICA th

Istituto Nazionale df Phies Nucloare

Serione do Pive 


\title{
Tests of a scintillating-fibre tracking detector based on position-sensitive photomultiplier readout
}

\author{
F. Bosi*, A. Cardini;, V. Cavasinni;, B. Di Girolamo; \\ S.I. Dolinsky ${ }^{\dagger} \quad$ V. Flaminio; $\quad$ A.M. Gorin \\ K.K. Kuroda, I.V. Manuilov\$, A. Penzo, D. Rizzi*; \\ R. Ruberti; A. A. Salnikov ${ }^{\dagger} \quad$ M. Tareb-Reyes $l$
}

\begin{abstract}
We report the results of measurements performed using a tracking detector based on $835 \mu \mathrm{m}$ scintillating fibres read-out by two positionsensitive photomultipliers. A six-layer hodoscope consisting of $1.2 \mathrm{~m}$ long fibres has been built and tested using cosmic rays. Subsequentely, two such hodoscopes have been assembled and tested using a hadron beam at the CERN PS. The efficiency per plane is found to be about $70 \%$ and the point resolution about $130 \mu \mathrm{m}$. The signal-to-noise ratio is about 2.4 and is dominated by crosstalk in the position-sensitive photomultipliers.
\end{abstract}

Submitted to Nuclear Instruments and Methods in Physics Research

* Dipartimento di Fisica, Università di Pisa and Istituto Nazionale di Fisica Nucleare, Sezione di Pisa, Italy

IINP, Novosibirsk, 630090, Russia

IHEP Protvino, Moscow region, 142284, Russia

§LAPP/IN2P3, Annecy-le-Vieux, France

IIstituto Nazionale di Fisica Nucleare, Sezione di Trieste, Italy

"LAPP/IN2P3, Annecy-le-Vieux, France 


\section{Introduction}

Tracking detectors based on scintillating fibres or liquid-scintillator filled glass capillaries have been investigated by many authors over the last few years $[1,2,3,4]$. The relatively small light-yield of plastic scintillating fibres and the only slightly better yield of glass capillaries filled with liquid scintillator $[2,10,11,12]$ place severe constraints on the light detectors used for readout. The most commonly used light detectors are the position-sensitive photomultipliers (PSPM), whose quantum efficiency ranges between 10 and $20 \%[5,6,7,8]$.

Different types of photomultipliers, based either on fine-grid mesh dynode structures, on microchannel plate or on foil dynode multiplication, exhibit distinctly different behaviours in their rate capability and crosstalk between neighbouring channels. They offer the common advantage of simplicity, fast time response and easy availability. The only seriously considered alternative, based on the solid state photomultipliers or visible light photon counters (VLPC) developed by the UCLA group $[4,9]$ in collaboration with Rockwell, offers a much better efficiency at the price of a substantially more complicated usage, due to the low temperature (about 6.5-7 K) at which these devices work.

The present study aims at assessing the ultimate performance that may be obtained, under realistic beam conditions, using the more appropriate, for our purpose, available scintillating fibres in association with a commercially available PSPM, the Philips XP1724A [13].

\section{The detector}

\subsection{Scintillating-fibre ribbons}

Commercially available fibre ribbons appropriate for the adopted type of PSPM were chosen in the construction of the tracking detector: the fibre type was Kuraray SCSF-38, whose spectral response peaks in the blue ( $\lambda=$ $430 \mathrm{~nm}$ ). The diameter of the plastic fibres is $0.835 \mathrm{~mm}$.

The fibre characteristics were measured in our laboratory using the methods described in [3] with the following results:

Attenuation length $l_{\text {att }}=200 \mathrm{~cm}$

Number of photoelectrons extrapolated at zero distance $N_{0}=3.6$ p.e.

Decay time $\tau=3.7 \mathrm{~ns}$ 

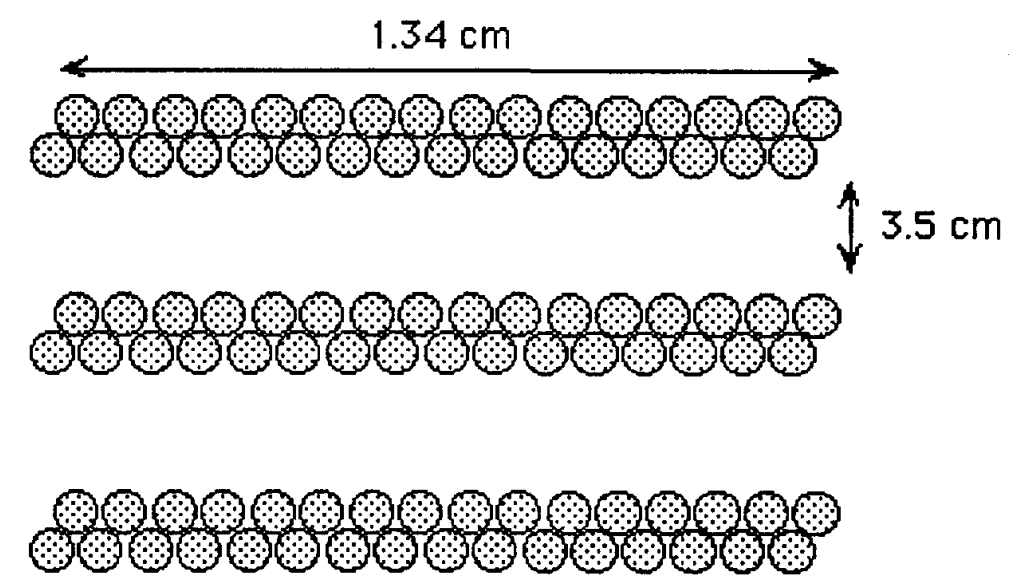

Figure 1: Detector geometry (not to scale)

This type of fibre is the most appropriate for a relatively short detector, as in our case $(1.2 \mathrm{~m})$, providing a satisfactory attenuation length and a high light yield. The other choice offered by Kuraray is the fibre SCF81, that has a longer attenuation length, but a slightly lower light yield and is suitable for detectors longer than $2.5 \mathrm{mu}$ [14].

Two separate hodoscopes were built, each comprising 3 superlayers of scintillating fibres. Each superlayer consisted of two single layers with 16 fibres each, staggered by half a fibre-diameter (figure 1). Each hodoscope had thus 96 fibres, that were read out by a 96 channel Philips PSPM.

A specially designed mask, having 96 holes of $0.835 \mathrm{~mm}$ in diameter at the locations of the pads on the PSPM input window was built. The fibre ends were glued to this mask, then the mask was polished and put in dry contact with the PSPM input window. The precise positioning of the mask is possible thanks to reference holes on the photomultiplier insulating shell provided by the firm for such purpose.

\subsection{The Philips XP1724/A PSPM}

The main characteristics of this PSPM [13] are:

- fibre optic window;

- useful photocathode diameter: $30 \mathrm{~mm}$;

- foil dynode structure; 


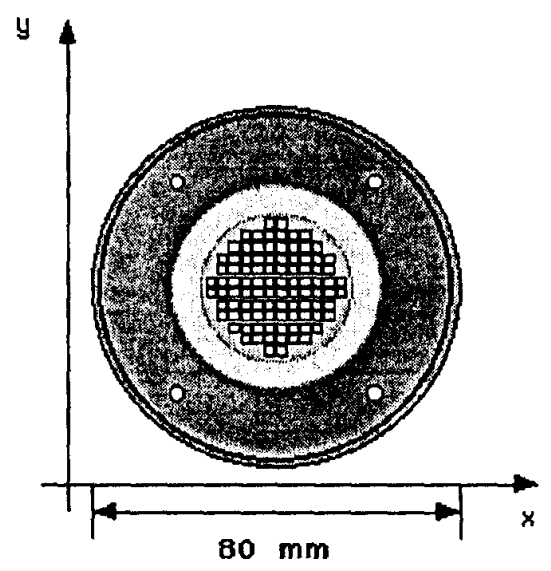

Figure 2: The PSPM input window; the anode matrix has the same geometry.

- last dynode arranged into 96 separate readout channels;

- pad dimensions: $2.54 \times 2.54 \mathrm{~mm}^{2}$; pad pitch: $2.54 \mathrm{~mm}$

- 10 dynode stages;

- gain of $10^{6}$ at $1.3 \mathrm{kV}$;

- quantum efficiency in the blue region $(420 \mathrm{~nm}): 18 \%$;

- fast rise time: 5 ns.

The last dynode is arranged as in figure 2.

\subsection{The readout}

The PSPM was operated with a resistor-chain arrangement such that the roles of the last dynode and of the anode were interchanged compared with its normal operation. Signals from the PSPM's were fed to shaping amplifiers located in the same box housing the PSPM. An amplification circuit was used to read out each output channel. The PSPM output pins were arranged in four quadrants and for each quadrant a card containing 24 amplifiers was used. Harris HA-2540 operational amplifiers were used in an inverting configuration. These operational amplifiers were chosen because of their large gain-bandwidth product $(400 \mathrm{MHz})$ and very high slew rate $(400 \mathrm{~V} / \mu \mathrm{s})$. The chosen gain of 80 implies a signal rise time of 70 ns. To decrease the heating 
in the PM housing (an aluminium box), due to the power dissipation of the circuit, an amplifier supply voltage of $\pm 8 \mathrm{~V}$ was chosen (a reduced value compared to the nominal $\pm 10 \mathrm{~V}$ ).

The second of the two hodoscopes built used a slightly modified version of the same amplification circuit, with a shorter shaping time.

After amplification the signals were transmitted through 15 meter long cables to the CAMAC crates housing the ADCs. The digitized information from all 192 channels was written to tape.

\section{Uniformity and crosstalk of the PSPM}

Uniformity of response across the PSPM input window and crosstalk between channels are of great importance in the present application. Therefore, detailed laboratory measurements of these parameters were carried out.

In the Philips specifications for this PSPM the following values are quoted:

Output sensitivity uniformity

- over 96 channels: maximum 10:1

- over at least 80 channels: maximum 5:1

Crosstalk between two adjacent channels: $5 \%$

The photocathode uniformity was measured by illuminating each channel by means of a $0.835 \mathrm{~mm}$ clear fibre glued to an LED. The other end of the fibre was glued to a mask and polished, with the same technique used in the production of our hodoscopes.

The system allowed to move the mask along the PSPM front window using two high precision screws and to measure the signal from different output channels.

The results of the measurements are shown in figure 3 . It is found that for $95 \%$ of channels the ratio of the maximum to the minumum signal amplitude is about 4 . This is in agreement with the Philips specifications for this type of PSPM.

The same measurement gives us an evaluation of crosstalk, that is related to the ratio of the average signal amplitude from neighbouring pads to that from the pad in contact with the clear fibre connected to the LED. A separate study of crosstalk along the $\mathrm{x}$ and the $\mathrm{y}$ direction (see figure 2) has been performed. To reduce the effect on the measurements of a possible misalignement of the clear fibre with respect to the PSPM channel, we have 


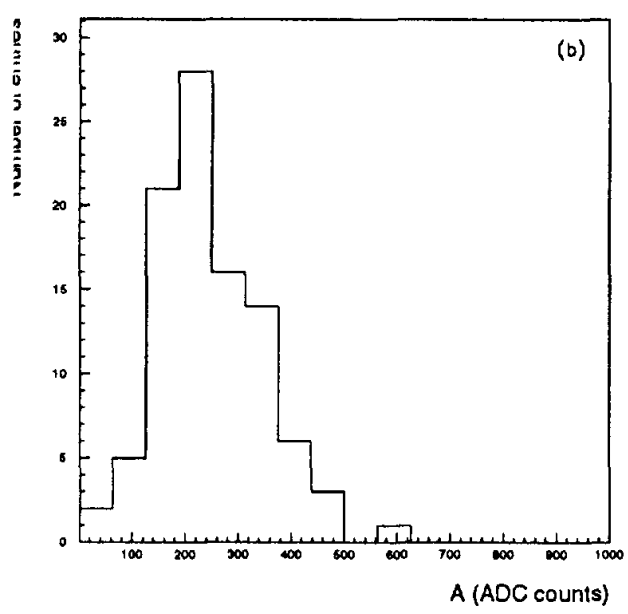

Figure 3: Distribution of signal amplitudes from pads of the Philips XP1724/A PSPM when the photocathode is uniformly illuminated by an LED.

used a slightly different definition of crosstalk: we take the ratio between the sum of the pulse-heights measured in the adjacent channels (up+down or left+right) and the sum of pulse-heights measured in the four adiacent channels plus the one under study. Figure 4(a) and Figure 4(b) show the distribution of the size of the crosstalk along the two directions separately.

In the vertical direction the crosstalk is on average $5 \%$, in agreement with the Philips specification, while in the other direction it equals $15 \%$ and for several channels it reaches $30 \%$. We have verified, by studying the correlations in pulse-height between neighbouring channels, that the crosstalk has its origin in the last dynode multiplication stages, rather than at the input window or at the first dynode stages. 

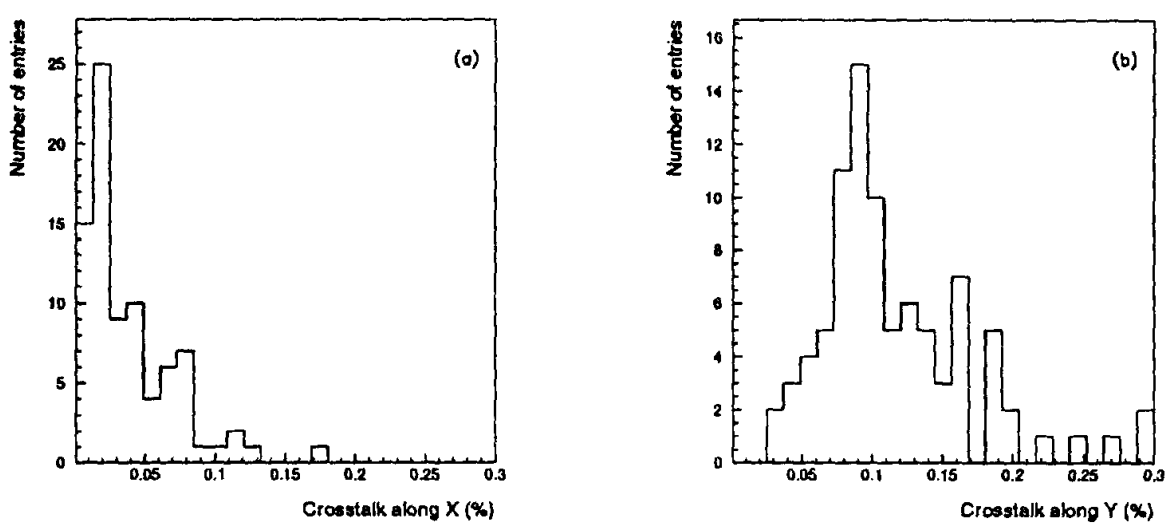

Figure 4: Distribution of the crosstalk for the Philips PSPM XP1724/A in the $x$ direction (a) and in the $y$ direction (b) for all pads. 


\section{Cosmic ray measurements}

A preliminary test of the hodoscope \# 1 was carried out in our laboratory using cosmic rays. The experimental setup is shown in figure 5 . The signals from two $30 \mathrm{~cm}$ long finger-counters, one above the hodoscope and the other under it, were put in coincidence. Each of the two finger-counters was viewed by a fast Hamamatsu R1635-02 photomultiplier [15].

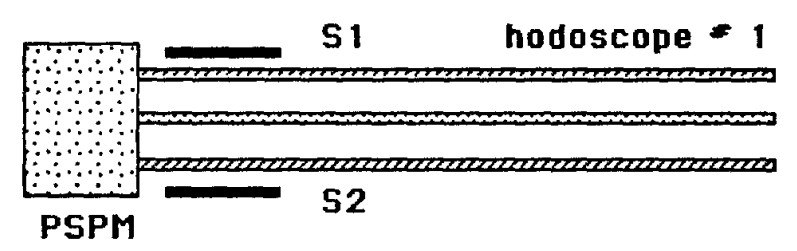

Figure 5: Sketch of the experimental setup for the cosmic ray.

\subsection{Event selection and reconstruction.}

Because of the presence of crosstalk in the PSPM a primary hit is usually accompanied by signals in nearby PSPM channels and appears thus as a cluster of pads. Different approaches have in the past been used to reduce the effects that such spurious hits produce on track reconstruction, in particular in the case of many-hit events, whose clusters may partly overlap. Among such approaches we may mention the use of the centre-of-gravity of pads, the use of the pad with largest pulse-height in a group of neighbouring pads and the fitting algorithm.

The procedure we have adopted consists in rejecting a hit if its pulseheight is lower by a given factor than the sum of those of the neighbouring ones in the PSPM (isolation cut). After this, track finding is performed by building all possible track candidates for any given pair of hits in different planes and selecting among these candidates the one that has the largest number of associated hits. A hit is considered as belonging to the track if its distance from the track is smaller than one fibre diameter. Following this, a straight line is fitted by minimizing the sum of square distances of associated hits from the track.

Before evaluating the efficiency and space resolution and after the isolation cut, a further cut was applied, requiring at least three aligned hits in different layers. The fraction of events rejected by this cut is $\sim 21 \%$, of which $\sim 11 \%$ are due to random trigger coincidences. This may be seen 

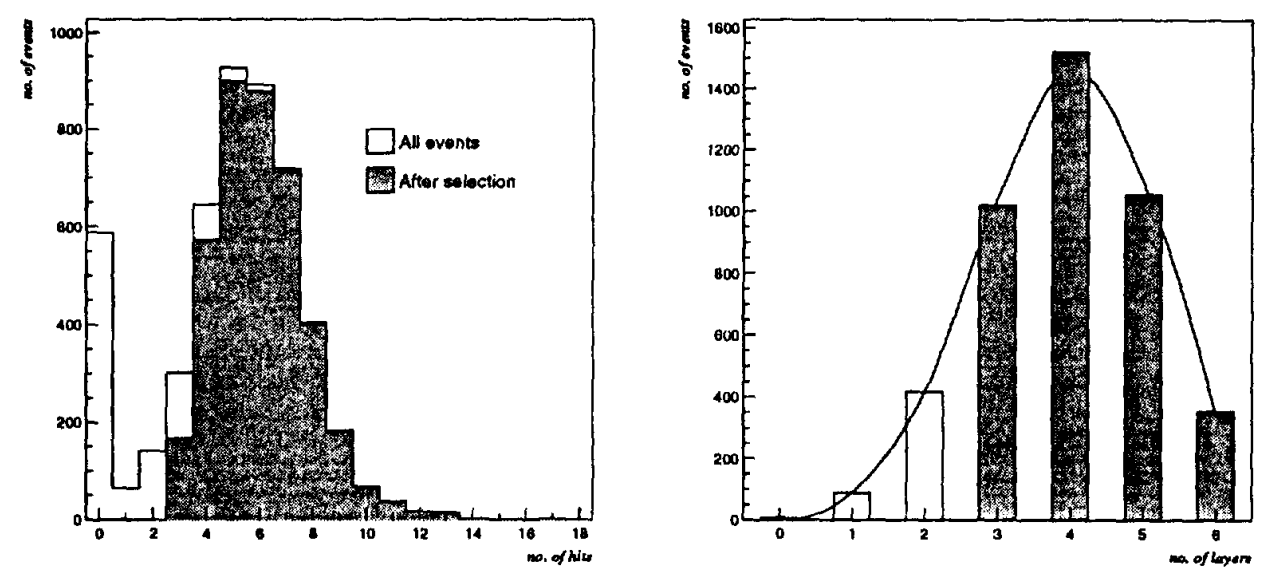

Figure 6: Number of hits per event (a) and number of layers which have hits near track (with selection) (b).

in figure 4.1, which shows the distribution of the number of hits per event. The peak at zero corresponds to the random triggers. The remaining $10 \%$ of events is consistent with the expected number of events having less than three hits on the track, for the value of efficiency found. The shaded part of the histogram in figure 4.1 represents the experimental distribution of the number of layers with hits on track obtained from cosmic ray data. The unshaded part is rejected by the algorithm. Fitting this with a binomial distribution (shown by the continuous curve) we obtain a mean efficiency of about $65 \%$ and a $10 \%$ probability for an event to have less than three hits. This leads to a global track reconstruction efficiency of $89 \%$.

Figure 7(a) shows the picture of an event with the track position reconstructed using the algorithm described above. Figure $7(\mathrm{~b})$ shows the same event as it appears on the PSPM photocathode.

In figure 8 we show for each layer the total number of hits, the number of hits associated to the track and the number of unassociated hits.

Figure 9 shows the efficiencies for each fibre layer calculated from cosmicray data. The average value found is about $65 \%$. It may be seen that the second layer has a much lower efficiency than the remaining ones. This is explained by a misalignement of the corresponding holes in the mask used to position the fibres on the PSPM front window.

In the following, a pair of nearby hits in different layers of one superlayer will be denoted a "doublet". All remaining hits will be denoted "singlets". 

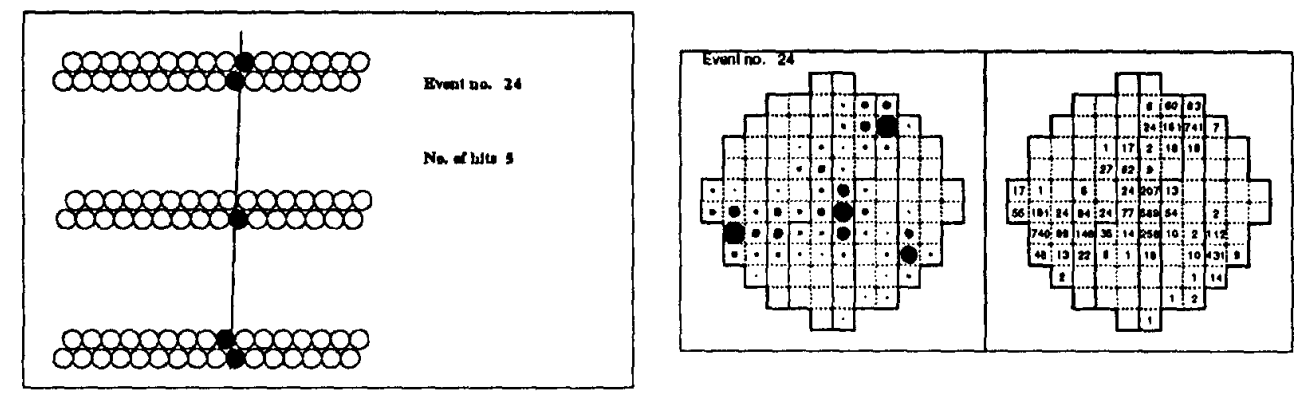

Figure 7: Example of a cosmic ray event. (a) View of hodoscope and track (not to scale). (b) View of the PSPM input window (the numbers are proportional to the pulse height).

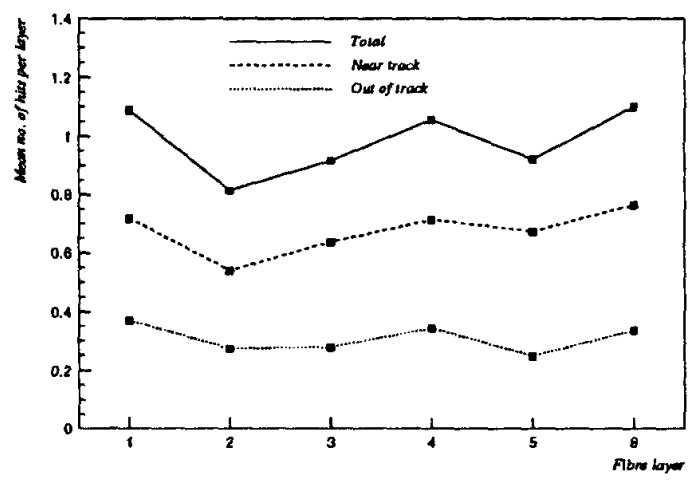

Figure 8: Number of hits per layer.

The position of a doublet hit is taken to be the mean position of the corresponding fibres. Figures 10(a) and (b) show the distribution of distances from the reconstructed track for singlet and doublet hits respectively. Figure 11 shows the combined distribution.

One can try to evaluate the resolution expected from the fibre geometry, assuming the position uncertainty for a single fibre of diameter $D$ to be given by $\sigma_{s}=D / \sqrt{12} \simeq 240 \mu \mathrm{m}$ and the uncertainty for a doublet to be $\sigma_{d}=\sigma_{s} / 2 \simeq 120 \mu \mathrm{m}$. Thus the global point resolution can be estimated as:

$$
\sigma_{t o t}=\frac{\sqrt{\bar{N}_{s} \sigma_{s}^{2}+\bar{N}_{d} \sigma_{d}^{2}}}{\bar{N}_{s}+\bar{N}_{d}}
$$




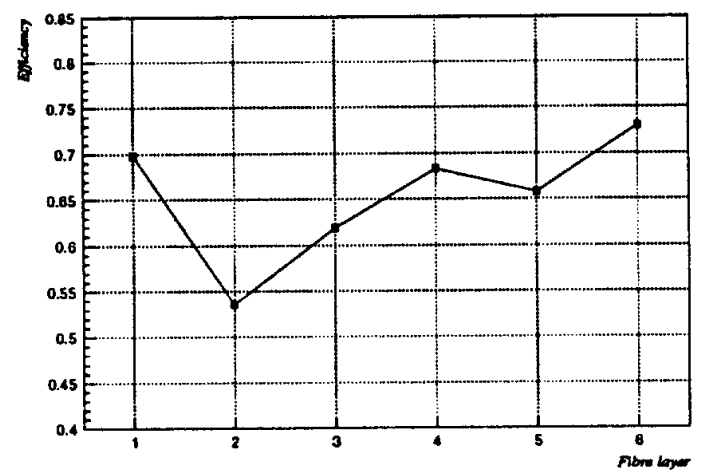

Figure 9: Efficiencies per layer for cosmic-ray data.

where $\bar{N}_{\text {, and }} \bar{N}_{d}$ are respectively the mean number of singlet and doublet hits in one event.

Using the experimental numbers one obtains a value for $\sigma_{\text {tot }}$ of about $120 \mu \mathrm{m}$. The value one obtains for the space resolution, fitting a gaussian to the central part of the distribution in figure 11 , is $140 \mu \mathrm{m}$. 

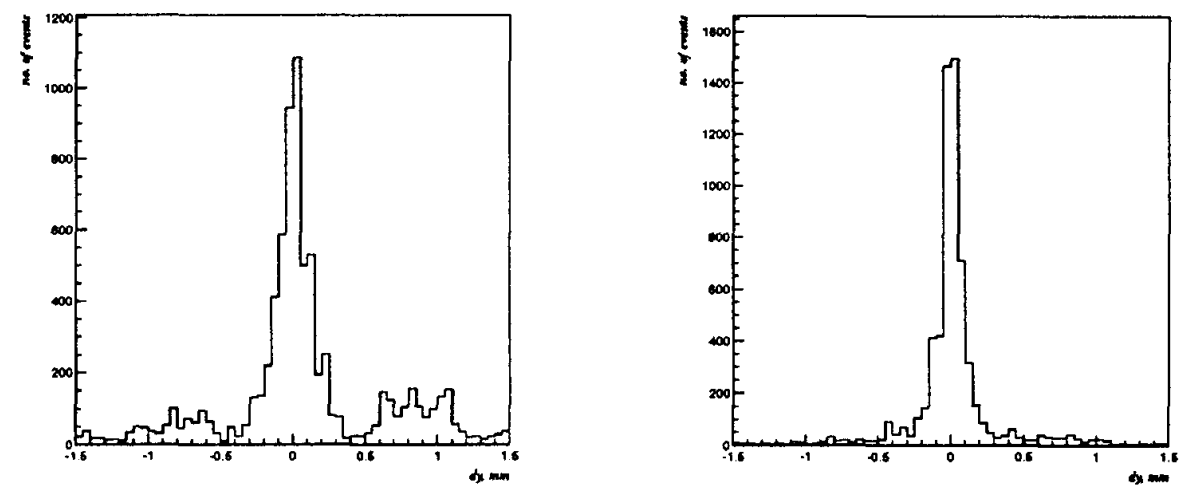

Figure 10: Distribution of hit distances from track. (a) singlets, (b) doublets.

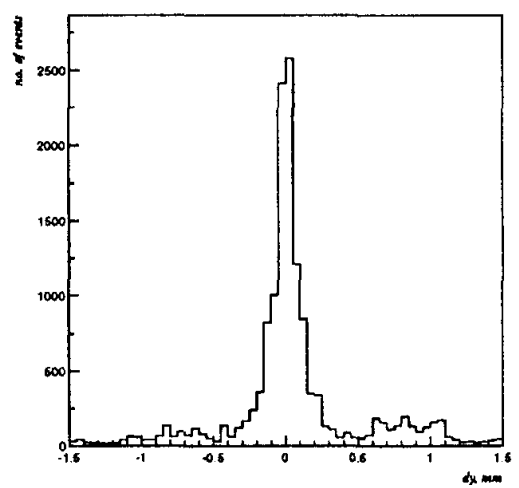

Figure 11: Distribution of hit distances from track (combined). 


\section{The test-beam setup}

Two hodoscopes like the one tested with cosmic rays were built and installed at the T7S test beam at the CERN PS. Figure 12 shows the setup used. The signals of scintillating counters B3 and B4, put in coincidence, were used for triggering on beam particles. A $1 \mathrm{~mm}$ thick movable counter $\mathrm{S} 1$ could be used to select particles in a narrow beam at a given transverse (vertical) position. The distance between the two hodoscopes was about $40 \mathrm{~cm}$.

Light, produced by scintillating fibres in the hodoscopes \# 1 and \# 2, was collected to the surface of two Philips PSPM's. 192 channels were thus used in this setup.

Event readout was performed using a data acquisition system based on a CES VIC system in VME and a SUN SPARCstation computer. Events were written on disk and subsequently unloaded on exabyte cartridges. A total of 200,000 events were collected, using different PSPM high voltage conditions and different geometries for the trigger counters.

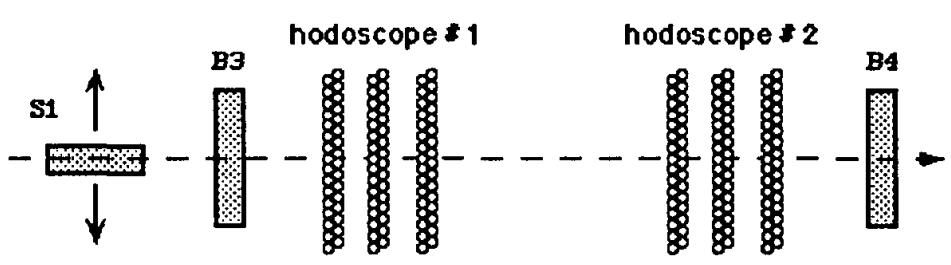

Figure 12: Sketch of the experimental setup at the test beam.

\section{Data analysis and results}

\subsection{Track finding.}

The track-finding algorithm was the same as the one used for the cosmic-ray data analysis; the only additional requirement applied was: at least two hits in two different layers for each hodoscope. The fraction of events rejected by this cut is $8.4 \%$. A typical example of reconstructed track is shown in figure 13 


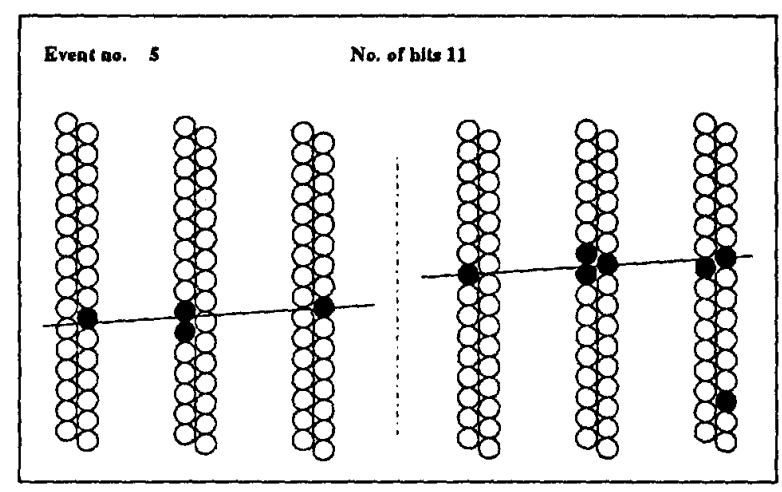

Figure 13: Beam event with reconstructed track (not to scale)

\subsection{Adjustment of fibre positions.}

During the track reconstruction procedure the distance between hit positions and reconstructed track for each layer of fibres was computed. The results showed that some layers had a systematic shift perpendicular to the beam axis. This was originated by a mechanical misalignement of the two hodoscopes. To correct for this effect an offset was computed for each plane using the reconstructed tracks.

\subsection{Efficiency.}

The average efficiency of fibre layers may be computed, assuming a binomial distribution, from the distribution of the number of layers with trackassociated hits. Figure 14 shows this distribution for one of the runs. From either the mean value or the width of this distribution one obtains a value of $70 \%$ for the efficiency.

The efficiency of individual layers may also be obtained as the ratio of the number of events with a track-associated hit on the given plane and the total number of reconstructed events. The parameters that mostly affect the efficiency are: the pulse-height threshold used and the crosstalk suppression level. A systematic analysis of the effect of these parameters on the efficiency has been carried out. The final choice used was a cut at 5 ADC counts above pedestal and a crosstalk suppression level of $35 \%$. Figure 15 shows the number of hits per layer, both in and out of track and both for beam and cosmic-ray data. Figure 16 shows the efficiency of each layer for both sets of data. 


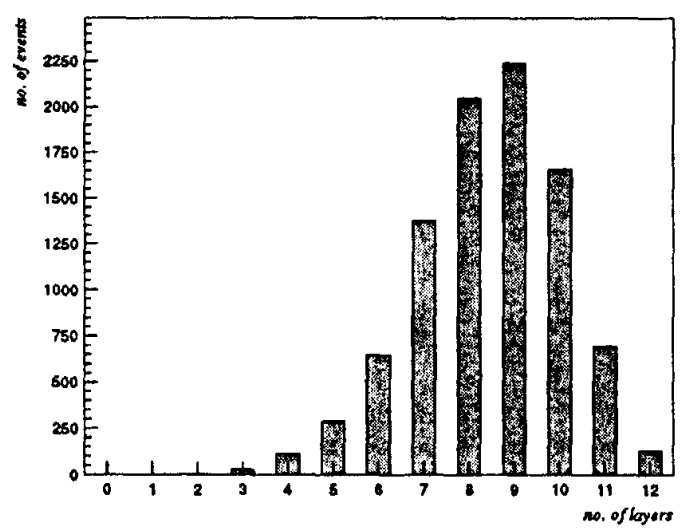

Figure 14: Distribution of the number of layers with hits.

Table 1 shows how the average efficiency changes using different combinations of crosstalk and ADC cuts.

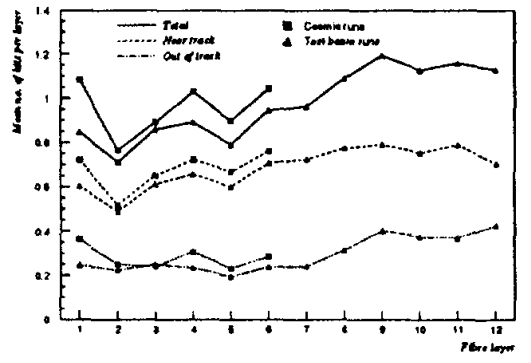

Figure 15: Mean number of hits per layer both for beam and cosmic-ray data.

We find that the first six fibre layers have lower efficiencies. This may be explained by a worse quality of the mask connecting the fibres to the PSPM input window in the older of the two hodoscopes. We notice also a difference of efficiency between cosmic ray and test beam data for the same hodoscope, that we attribute to a worse optical contact in the test beam setup. 


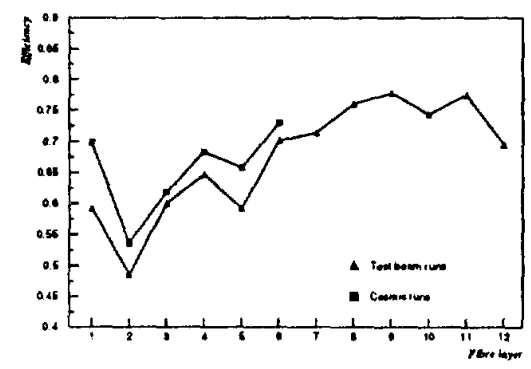

Figure 16: Efficiency per layer for beam and cosmic-ray data.

\begin{tabular}{|c|c|c|c|c|}
\hline $\mathrm{ADC} / \mathrm{cs}$ & efficiency & hits on track & hits out of track & hits total \\
\hline $5 / 25$ & 0.679 & 0.696 & 0.364 & 1.060 \\
$5 / 35$ & 0.673 & 0.683 & 0.293 & 0.976 \\
$5 / 50$ & 0.661 & 0.666 & 0.223 & 0.889 \\
$10 / 35$ & 0.664 & 0.673 & 0.270 & 0.943 \\
$20 / 35$ & 0.645 & 0.654 & 0.245 & 0.899 \\
\hline
\end{tabular}

Table 1: Mean efficiency per layer and number of hits for different choices of ADC and crosstalk-level cuts (cs)

The efficiency obtained from this measurement, averaged over all planes, is equal to $67 \%$, which is in agreement with the values obtained via the binomial distribution.

\subsection{Space resolution.}

We define as space resolution the sigma obtained by fitting a gaussian to the distribution of distances of hits from the track. A typical distribution of distances is shown in figure 17, where a superposition of two gaussians has been used to fit the data. The sigma of the main distribution is about $130 \mu \mathrm{m}$.

Possible sources of the observed background is residual crosstalk or slightly incorrect positioning of fibres on the photocathode of the PSPM. This effect is clearly seen for fibres of the second layer in the first hodoscope, which show an additional peak in the distribution at a position equivalent to ten fibres from the track. Additional effects which may contribute to background are 


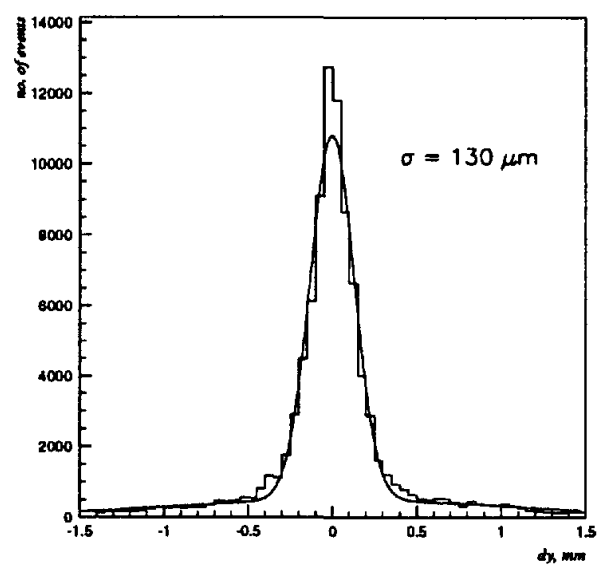

Figure 17: Distribution of adjusted hits positions.

delta rays and events with two or more tracks.

\subsection{Angular resolution.}

The distribution of angles for the reconstructed tracks is shown in figure 18 for one of the runs. This has been fitted using a gaussian. Its sigma is 0.043 degrees. The value found may be compared with the one expected from the detector resolution, that is estimated to be about 0.03 degrees. The difference between these two values may be due to multiple scattering in the material upstream our detector.

\subsection{Beam impact position.}

During the test-beam run, no external tracking detectors were available. For a qualitative evaluation of the absolute space precision the knowledge of the position of the external trigger counter $S 1$ was used. In three subsequent runs this counter was positioned at three different heights, separated approximatevely by $3 \mathrm{~mm}$. The distributions of the reconstructed beam positions for these three runs are shown in figure 19. The distance between the mean values is found to be $3.2 \mathrm{~mm}$ and $3.1 \mathrm{~mm}$ in the two cases, in agreement with the expected value. 


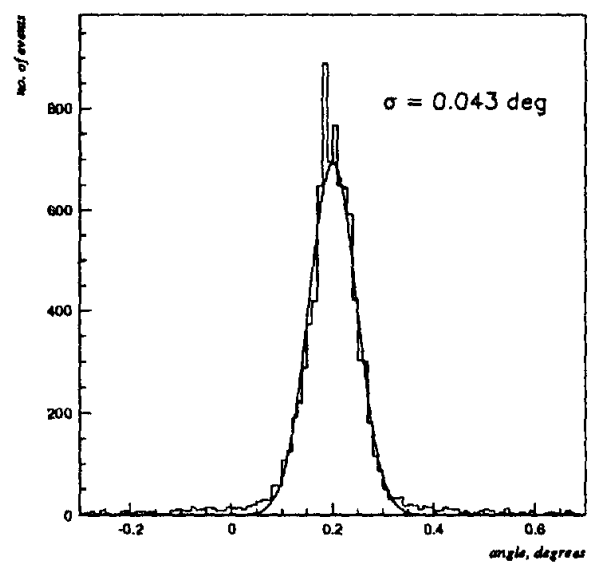

Figure 18: Angular distribution of reconstructed tracks.

\subsection{Montecarlo simulation}

A simulation of the experimental setup has been carried out, in order to verify the consistency of our results and the quality of our selection and reconstruction procedure. The input values for the efficiency (67\%) and for the fraction of random hits $(29 \%)$ were those found in the test-beam data. Crosstalk was also taken into account in the simulation, using the distributions obtained from the real data. Incident beam particles were generated with a uniform space distribution and a gaussian angular shape. The average angle was the one found in the test-beam data and the sigma was 0.03 degrees. The generated events underwent the same filtering and reconstruction procedure as the test-beam data. Figure 20 compares the distribution of distances of hits from tracks as found in Montecarlo and in real data.

A study has been made of differences between generated and reconstructed track parameters. In particular an analysis has been made of a) the difference in angle between generated and reconstructed tracks and $b$ ) the difference in position between them, computed half way between the first and last fibre layer. Figure 21 shows the lego plot of the angular difference vs the space distance. The angular distribution peaks at zero and its sigma is 0.027 degrees. The distribution of space distances also peaks at zero and its sigma is $90 \mu \mathrm{m}$. In the two-dimensional distribution only $4.5 \%$ of events fall outside 3 sigma from the centre. We conclude that the selection and reconstruction procedure does not introduce any bias or distortion in the data. 


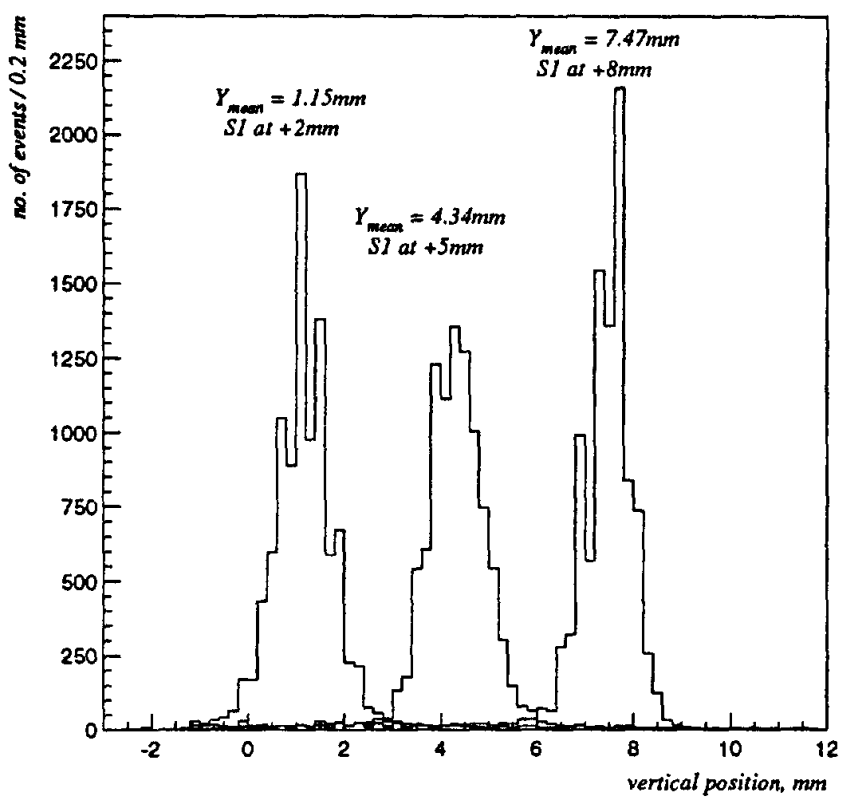

Figure 19: Position of the impact point of reconstructed tracks for three different runs described in the text 


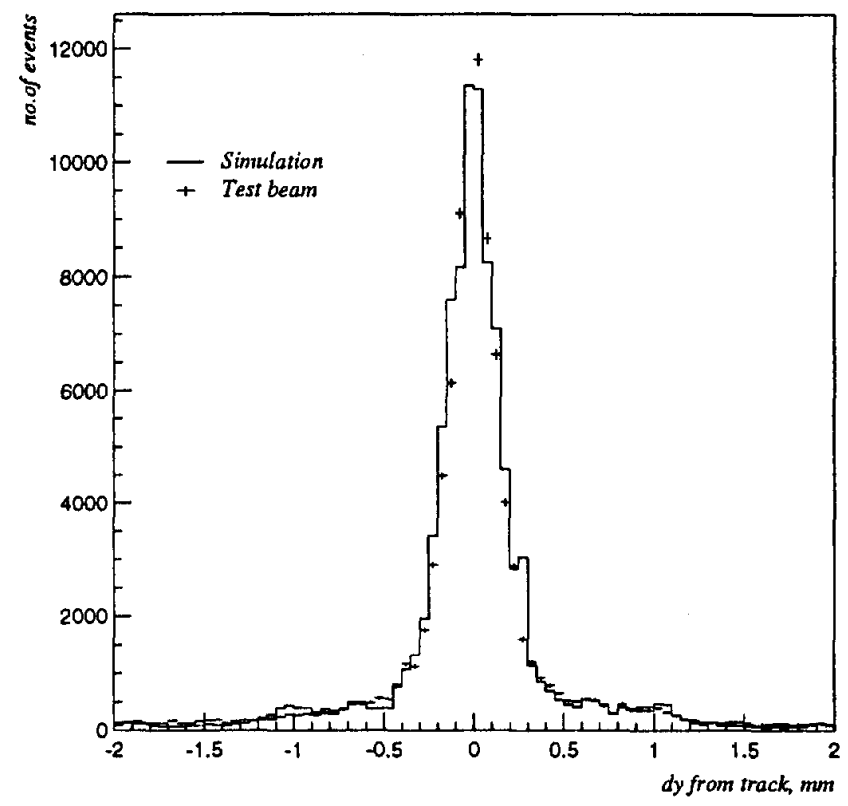

Figure 20: Distribution of distances of hits from tracks for simulated (solid line) and test-beam data (crosses) 


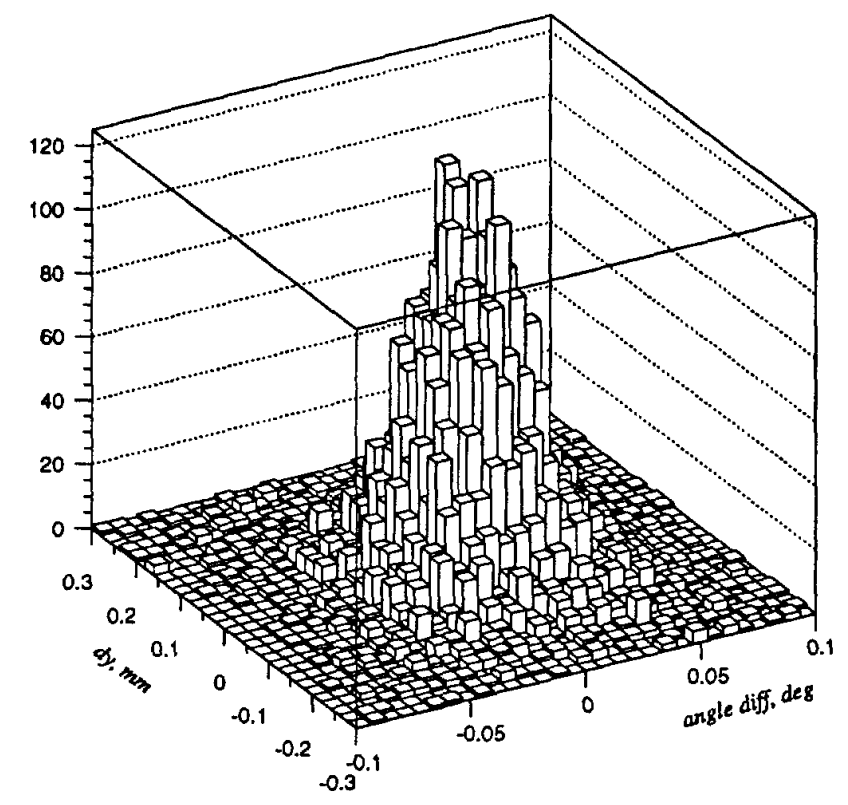

Figure 21: Lego plot of angle difference between generated and reconstructed tracks vs space distance between the two 


\section{Conclusions}

Two hodoscope counters, consisting each of a $1.2 \mathrm{~m}$ long array of 96 scintillating optical fibres read-out by a Philips XP1724/A position-sensitive photomultiplier, have been built and tested using cosmic rays and a high energy particle beam ( $7 \mathrm{GeV}$ ) from the CERN proton-synchrotron. In each hodoscope the fibre ribbons were arranged in 3 different superlayers of two layers each. Each PSPM was read-out using amplifiers followed by 96 ADC channels for each hodoscope. A pattern recognition algorithm has been developed to reconstruct tracks in the hodoscope, eliminating spurious signals due to crosstalk in the PSPM. The efficiency found amounts to about $70 \%$ per fibre layer and the space resolution is about $130 \mu \mathrm{m}$. A Montecarlo simulation of the detector shows that the track-finding algorithm does not introduce any bias and that the intrinsic space precision of the hodoscope is approximately $90 \mu \mathrm{m}$.

\section{Acknowledgements}

Thanks are due to our colleagues of the RD17 collaboration: V. Agoritsas, J. Dufournaud, V. Rykalin and D. Sillou for the help during the data taking. We wish to acknowledge the invaluable help of the administrative and technical staff of the Pisa INFN laboratory, and in particular of G. Favati, M. Favati, E. Pucciarelli and P. Villani. We also wish to thank the laboratory Director, G.M. Pierazzini for his continous support. 


\section{References}

[1] M. Adinolfi et al., Nucl. Instr. and Meth. A315 (1992) 177

[2] S. Golovkin et al., Nucl. Instr. and Meth. A305 (1991) 385

[3] D. Autiero et al., Nucl. Instr. and Meth. A336 (1993) 521

[4] M. Atac et al., Nucl. Instr. and Meth. A314 (1991) 56

[5] K. Kuroda et al., Nucl. Instr. and Meth. A300 (1991) 259

[6] S. Majewski et al., Nucl. Instr. and Meth. A323 (1992) 489

[7] J. Bähr et al., Nucl. Instr. and Meth. A324 (1993) 145

[8] A. Cardini et al., Preprint INFN PI/AE 94/08

[9] B. Abbott et al., Nucl. Instr. and Meth. A327 (1993) 319

[10] A. Cardini et al., Nucl. Instr. and Meth. A346 (1994) 163

[11] A. Cardini et al., Preprint INFN PI/AE 94/12

[12] C. Cianfarani et al., Nucl. Instr. and Meth. A339 (1994) 449

[13] Philips Photonics, XP1700 photomultiplier series (1992)

[14] Kuraray Co., Ltd., Methacrylic Resin Division, Hatchobory 2-Chome, Chuo-ku, Tokyo 104, Japan

[15] Hamamatsu photonics K.K. Photomultiplier catalogue, February 1994 


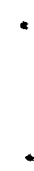

